\title{
Defining the role of bempedoic acid in lowering low-density lipoprotein cholesterol
}

\author{
Oleksii Korzh* \\ Department of General Practice-Family Medicine, Kharkiv Medical Academy of Postgraduate Education, Kharkiv, Ukraine.
}

\section{ARTICLE INFO \\ Received on: 27/07/2020 \\ Accepted on: 04/10/2020 \\ Available online: 05/12/2020}

\section{Key words:}

Bempedoic acid, ETC-1002,

LDL, statin, cardiovascular disease.

\begin{abstract}
There is an unmet medical need for patients who cannot achieve a sufficient reduction in low-density lipoprotein (LDL) cholesterol with the existing treatment options. Statin therapy remains the mainstay of treatment for both primary and secondary prevention. However, many patients cannot tolerate statin therapy because of statin-associated muscle symptoms. This underlines the importance of developing alternative cholesterol-lowering methods with good efficacy and tolerance in addition to statins. Bempedoic acid (ETC-1002) is one such novel cholesterol-lowering drug. It is an inhibitor of adenosine triphosphate citrate lyase, a cellular enzyme responsible for the production of precursors for the synthesis of fatty acids and cholesterol. Bempedoic acid reduces the synthesis of cholesterol in the liver cells and triggers the compensatory activation of the LDL receptor, as well as complementing other mechanisms targeted by current therapies, which leads to an additional decrease in LDL cholesterol. The current clinical trial's results suggest that bempedoic acid may represent a new therapeutic approach for lowering LDL cholesterol. The recent approval of bempedoic acid by the Food and Drug Administration (FDA) offers an additional option for lowering LDL cholesterol in patients with atherosclerotic cardiovascular disease or heterozygous familial hyperlipidemia. Additional data on the effects of bempedoic acid on long-term cardiovascular outcomes are currently being investigated in large cardiovascular outcome studies. In the present study, we discuss the history and development of bempedoic acid, the pharmacology, the relevant clinical trials, and the potential role of bempedoic acid as a lipid-lowering medication in the context of other currently available lipid-lowering therapies.
\end{abstract}

\section{Introduction}

Cardiovascular disease (CVD) is a major public health problem and the leading cause of mortality and morbidity worldwide (Arnett et al., 2019). Changes in the metabolism of lipids and lipoproteins play an important role in the pathogenesis of CVD. Numerous epidemiological studies and randomized clinical trials have shown that elevated low-density lipoprotein (LDL) cholesterol is the main cause of atherosclerotic CVDs (Koskinas et al., 2018; Navarese et al., 2018).

Management of serum cholesterol is the main task in preventing atherosclerotic cardiovascular events (Brinton,

"Corresponding Author

Oleksii Korzh, Department of General Practice-Family Medicne, Kharkiv Medical Academy of Postgraduate Education, Kharkiv, Ukraine.

E-mail: okorzh2007@gmail.com
2015; Jacobson et al., 2015; Khera et al., 2016; Martin et al., 2016). Currently, the standard treatment for patients with hypercholesterolemia is primarily statins, which can lower LDL cholesterol. Lowering LDL cholesterol with statins inhibits the progression of coronary atherosclerosis and reduces cardiovascular mortality and morbidity. However, some patients, especially patients with heterozygous familial hypercholesterolemia, coronary artery disease (CAD), risk equivalents of CAD, and other clinical manifestations of atherosclerotic CVD (Grundy et al., 2019), require an additional reduction in LDL cholesterol in addition to what can be achieved with a maximum tolerated statin therapy.

In addition, there are patients who cannot tolerate statins due to adverse effects, such as muscle pain or an increase in blood glucose. This underlines the importance of developing alternative cholesterol-lowering methods with good efficacy and tolerance in addition to statins (Rosenson et al., 2017). 
Managing LDL cholesterol can also be achieved by other mechanisms, such as inhibiting intestinal cholesterol absorption [i.e., ezetimibe (EZE)] or preventing degradation of LDL receptors (i.e., PCSK9 inhibitors) (Table 1). It is important to note that each of these mechanisms primarily reduces LDL cholesterol by upregulating the activity of LDL receptors, a mechanism that has been shown to reduce cardiovascular events (Bonaca et al., 2018; O'Donoghue et al., 2019). These strategies significantly influenced further expectations of lowering the cardiovascular risk in patients with concomitant high-risk diseases, which led to the combination of statins with other existing drugs that lower LDL cholesterol and to the need for new treatments with mechanisms that complement the effects of statins without increasing side effects in skeletal muscle (Newman et al., 2019).

Bempedoic acid (BemA or ETC-1002) is one such novel cholesterol-lowering drug. It is an inhibitor of adenosine triphosphate (ATP) citrate lyase (ACLY), a cellular enzyme responsible for the production of precursors for the synthesis of fatty acids and cholesterol. ETC-1002 effectively reduces LDL and apolipoprotein (apo) B-containing lipoproteins (Bilen and Ballantyne, 2016; Pinkosky et al., 2013).
ETC-1002 was first discovered at the original Esperion Therapeutics, which was acquired by Pfizer in 2004, and then stood out as Esperion Therapeutics in 2008 along with ETC1002 and other assets. Esperion continues to develop ETC-1002, which is currently in phase III trials as an agent for lowering LDL cholesterol in patients with hypercholesterolemia.

Both phase 2 and phase 3 clinical trials showed that BemA as monotherapy or when added to background lipidlowering therapy significantly lowered LDL-C, as well as other relevant lipids and biomarkers (Ruscica et al., 2019).

The US Food and Drug Administration has approved BemA and the BemA and EZE-fixed dose combination tablet for the treatment of adults with heterozygous familial hypercholesterolemia or established atherosclerotic CVD who require additional lowering of LDL cholesterol. The recommended dosage for the new drug, in combination with maximally tolerated statin therapy, is $180 \mathrm{mg}$ administered orally once daily.

The applications to the European Medicines Agency for both preparations received positive opinions from the Committee for Medicinal Product for Human Use of the European Medicines Agency (EMA).

Table 1. Lipid modifying pharmaceuticals in development.

\begin{tabular}{|c|c|c|c|c|c|c|c|c|}
\hline Name & Company & Target & $\begin{array}{l}\text { Support for } \\
\text { Target }\end{array}$ & $\begin{array}{l}\text { Mechanism of } \\
\text { Action }\end{array}$ & Indication & Stage & $\begin{array}{l}\text { Biochemical } \\
\text { Effect }\end{array}$ & $\begin{array}{l}\text { Current or Possible } \\
\text { Use in } 5 \mathrm{y}\end{array}$ \\
\hline Lomitapide & Aegerion & MTP & $\mathrm{RD} / \mathrm{BCM} / \mathrm{AM}$ & $\begin{array}{l}\text { Oral MTP } \\
\text { inhibitor }\end{array}$ & $\mathrm{HoFH}$ & Approved & $\begin{array}{l}\text { Reduces LDL-C } \\
\text { and TG }\end{array}$ & $\begin{array}{l}\text { HoFH; apheresis- } \\
\text { eligible } \\
\text { hypercholesterolemia }\end{array}$ \\
\hline Mipomersen & IONIS/Genzyme & APOB & $\begin{array}{l}\mathrm{RD} / \mathrm{BCM} / \mathrm{AM} / \\
\mathrm{MR}\end{array}$ & $\begin{array}{l}\text { Anti-APOB } \\
\text { antisense }\end{array}$ & $\mathrm{HoFH}$ & Approved & Reduces LDL-C & $\begin{array}{l}\text { HoFH; apheresis- } \\
\text { eligible } \\
\text { hypercholesterolemia }\end{array}$ \\
\hline $\begin{array}{l}\text { AAV8.TBG.hLDLR } \\
\text { (RGX-501) }\end{array}$ & RegenXBio & LDLR & $\begin{array}{l}\mathrm{RD} / \mathrm{BCM} / \mathrm{AM} / \\
\mathrm{MR}\end{array}$ & $\begin{array}{l}\text { LDLR gene } \\
\text { theraphy }\end{array}$ & $\mathrm{HoFH}$ & Phase 1 & Reduces LDL-C & $\begin{array}{l}\text { HoFH because of } \\
\text { biallelic LDLR gene } \\
\text { mutations }\end{array}$ \\
\hline Bempedoic acid & Esperion & $\mathrm{ACL}$ & $\begin{array}{l}\mathrm{RD} / \mathrm{BCM} / \mathrm{AM} / \\
\mathrm{MR}\end{array}$ & $\begin{array}{l}\text { Oral ACL } \\
\text { inhibitor }\end{array}$ & Hypercholestereolemia & Phase 2-3 & Reduces LDL-C & $\begin{array}{l}\text { Adjunct to reduce } \\
\text { LDL-C in ASCVD } \\
\text { risk,FH,SI }\end{array}$ \\
\hline Gemcabene & Gemphire & ND & $\mathrm{BCM}$ & $\begin{array}{l}\text { Unknown; not } \\
\text { PPAR }\end{array}$ & $\begin{array}{l}\text { Hypercholestereolemia; } \\
\text { hypertriglyceridemia }\end{array}$ & Phase 2-3 & $\begin{array}{l}\text { Reduces LDL-C } \\
\text { and TG }\end{array}$ & $\begin{array}{l}\text { Adjunct to reduce } \\
\text { LDL-C in ASCVD } \\
\text { risk,FH,SI }\end{array}$ \\
\hline Inclisirian & Alnylam & PCSK9 & $\begin{array}{l}\mathrm{RD} / \mathrm{BCM} / \mathrm{AM} / \\
\mathrm{MR}\end{array}$ & $\begin{array}{l}\text { Anti-PCSK9 } \\
\text { ASO }\end{array}$ & $\mathrm{HeFH}$ & Phase 3 & Reduces LDL-C & $\begin{array}{l}\text { HeFH, high ASCVD not } \\
\text { at LDL-C target }\end{array}$ \\
\hline Alipogene tipavovec & uniQure & LPL & $\begin{array}{l}\mathrm{RD} / \mathrm{BCM} / \mathrm{AM} / \\
\mathrm{MR}\end{array}$ & $\begin{array}{l}\text { LPL gene } \\
\text { therapy }\end{array}$ & FCS & Approved & Reduces TG & $\begin{array}{l}\text { FCS because of biallelic } \\
L P L \text { ggene mutations }\end{array}$ \\
\hline Pradigastat & Novartis & DGAT $_{1}$ & $\mathrm{RD} / \mathrm{BCM}$ & $\begin{array}{l}\text { DGAT1 } \\
\text { 1inhibitor }\end{array}$ & $\begin{array}{l}\text { FCS; severe } \\
\text { hypertriglyceridemia }\end{array}$ & $\begin{array}{l}\text { No updates } \\
\text { since } 2017\end{array}$ & Reduces TG & Uncertain future \\
\hline Volanesorsen & IONIS/Akcea & APOC3 & $\begin{array}{l}\mathrm{RD} / \mathrm{BCM} / \mathrm{AM} / \\
\mathrm{MR}\end{array}$ & $\begin{array}{l}\text { Anti-APOC3 } \\
\text { ASO }\end{array}$ & FCS & Not approved & Reduces TG & Uncertain future \\
\hline Evinacumab & Regeneron & ANGPTL3 & $\begin{array}{l}\mathrm{RD} / \mathrm{BCM} / \mathrm{AM} / \\
\mathrm{MR}\end{array}$ & $\begin{array}{l}\text { Anti-ANGPTL3 } \\
\text { antibody }\end{array}$ & $\begin{array}{l}\text { Hypercholestereolemia; } \\
\text { hypertriglyceridemia }\end{array}$ & Phase 2-3 & $\begin{array}{l}\text { Reduces TG, } \\
\text { LDL-C, and } \\
\text { HDL-C }\end{array}$ & $\begin{array}{l}\text { FCS; HoFH; refractory } \\
\text { severe hyperlipidemia }\end{array}$ \\
\hline IONIS-APO(a)- $\mathrm{L}_{\mathrm{Rx}}$ & IONIS/Akcea & apo(a) & $\begin{array}{l}\mathrm{RD} / \mathrm{BCM} / \mathrm{AM} / \\
\mathrm{MR}\end{array}$ & $\begin{array}{l}\text { Anti-apo(a) } \\
\text { ASO }\end{array}$ & Elevated LP(a) levels & Phase 2-3 & Reduces Lp(a) & $\begin{array}{l}\text { Severe elevated Lp(a); } \\
\text { ASCVD, CAVD risk }\end{array}$ \\
\hline CSL-112 & CSL Behring & APOA1 & $\begin{array}{l}\mathrm{RD} / \mathrm{BCM} / \mathrm{AM} / \\
\mathrm{MR}\end{array}$ & $\begin{array}{l}\text { APOA1 peptide } \\
\text { infusion }\end{array}$ & Low HDL-C & Phase 3 & Raises HDL-C & $\begin{array}{l}\text { Severe depressed } \\
\text { HDL-C; ASCVD risk }\end{array}$ \\
\hline Sebelipase & Alexion & LIPA & $\mathrm{RD} / \mathrm{BCM} / \mathrm{AM}$ & $\begin{array}{l}\text { LIPA } \\
\text { replacement }\end{array}$ & LIPA deficiency & Approved & $\begin{array}{l}\text { Reduces TG, } \\
\text { LDL-C and } \\
\text { liver lipids }\end{array}$ & $\begin{array}{l}\text { LIPA deficiency or } \\
\text { CESD only }\end{array}$ \\
\hline $\begin{array}{l}\text { ACP-501/ } \\
\text { MEDI6012 }\end{array}$ & Medimmune & LCAT & $\mathrm{RD} / \mathrm{BCM} / \mathrm{AM}$ & $\begin{array}{l}\text { Recombinant } \\
\text { LCAT }\end{array}$ & LCAT deficiency & Phase 1 & $\begin{array}{l}\text { Raises HDL-C, } \\
\text { redistributes } \\
\text { HDL subfractions }\end{array}$ & $\begin{array}{l}\text { LCAT deficiency and } \\
\text { perhaps other HDL-C } \\
\text { deficiencies }\end{array}$ \\
\hline
\end{tabular}


Thus, in the present study, we discuss the history and development of BemA, the pharmacology, the relevant clinical trials, and the potential role of BemA as a lipid-lowering medication in the context of other currently available lipid-lowering therapies.

\section{Mechanism of action and pharmacology of bempedoic acid}

BemA (8-hydroxy-2,2,14,14-tetramethylpentadecanedioic acid) is a new drug that reduces cholesterol synthesis by inhibiting ACLY (Figure 1). In animal models, BemA lowers LDL cholesterol and inhibits fatty acid synthesis, but in humans, it is mainly used to lower LDL cholesterol (Nikolic et al., 2014). BemA significantly reduced LDL-C (up to $32 \%$ ) in patients with normal or elevated triglycerides (TG). This effect of lowering lipid levels is better than currently approved nonstatin-based lipid-lowering drugs and is similar to the usual doses of many statins (Lemus and Mendivil, 2015). BemA also lowers levels of apoB, LDL cholesterol (nonHDL cholesterol), and highly sensitive C-reactive protein (hsCRP) (Zagelbaum et al., 2019) and also has a beneficial effect on body weight and blood pressure (Nikolic et al., 2014). Most importantly, BemA is well tolerated by patients without significant side effects.

ACLY is an important enzyme in the cholesterol biosynthesis pathway upstream of the 3-hydroxy-3-methylglutaryl coenzyme A reductase [which is a target for statins (Ference et al., 2019)]. ACLY produces acetyl-CoA (AcCoA) from mitochondrial citrate for the biosynthesis of cholesterol and fatty acids (Burke and Huff, 2017). ACLY forms homotetramers through the C-terminus (citrate synthase homeodomain) to facilitate the binding of ACLY to CoA and AcCoA production (Bazilevsky et al., 2019). The Mendelian randomization of large human study cohorts has confirmed that ACLY can be used as a promising therapeutic target for lowering LDL cholesterol and protecting against atherosclerosis (Burke et al., 2019).

ACLY catalyzes the conversion of citric acid to oxaloacetate and AcCoA (Verschueren et al., 2019). AcCoA promotes key biochemical reactions, including the synthesis of fatty acids, cholesterol, and acetylcholine, as well as acetylation of protein substrates, including histones (Sivanand et al., 2017; Wellen et al., 2009). Typically, citrate is synthesized in mitochondria and transported to the cytosol via a mitochondrial citrate carrier protein (encoded by the SLC25A1 gene), which produces AcCoA and oxaloacetate by ACLY.

Recently, the crystal structures of bacteria and ACLY have been resolved with high resolution, in which conformational plasticity, substrate binding, and ACLY catalytic processes have

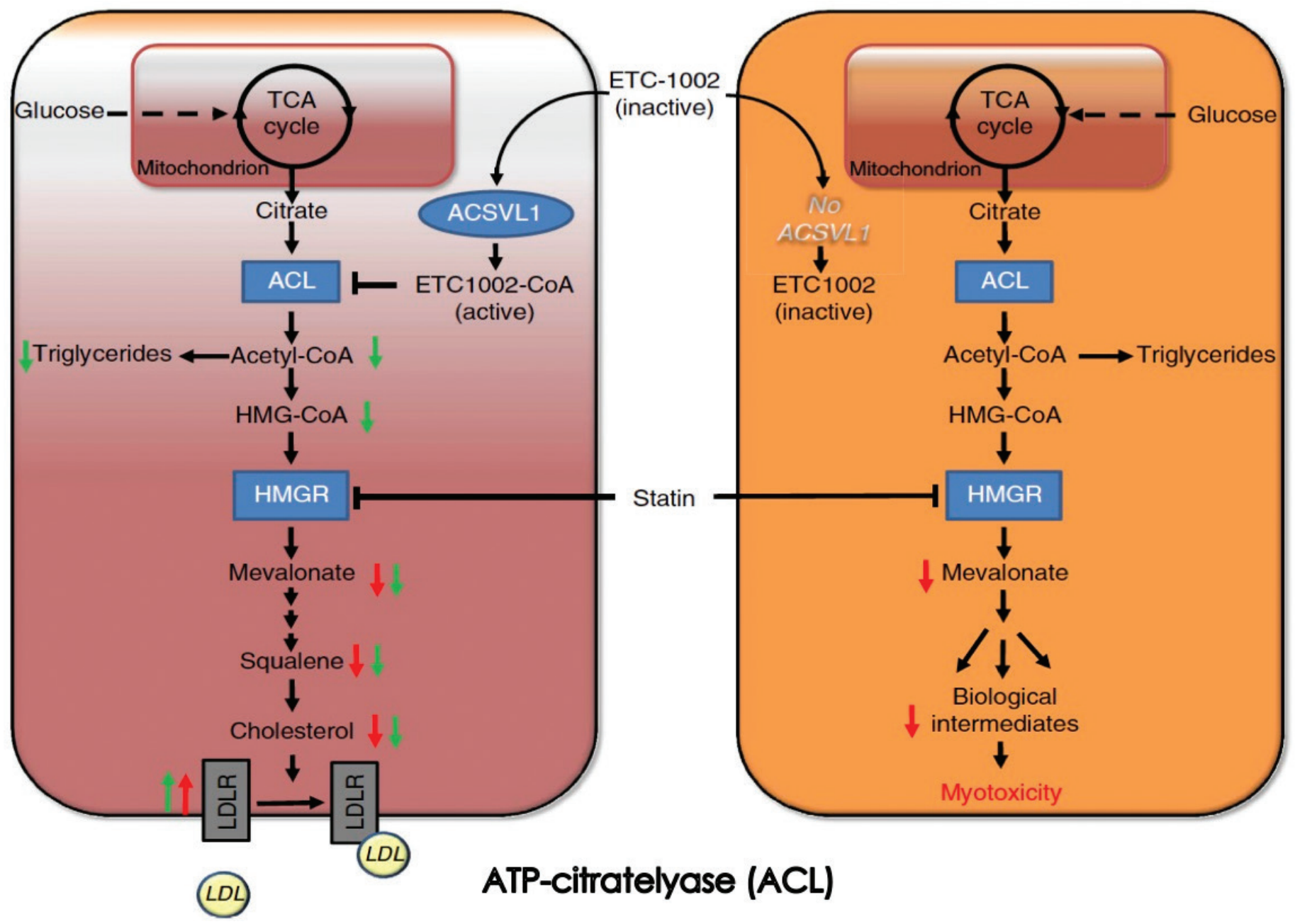

Figure 1. Major metabolic pathways affected by bempedoic acid (ETC 1002) in humans. Author's figure. No permission is required. 
been characterized. These results will provide important clues for the development of new ACLY inhibitors and highlight the therapeutic potential of ACLY inhibitors in hyperlipidemia and various types of cancer (Verschueren et al., 2019; Wei et al., 2019).

ACLY is a key enzyme in the production of AcCoA, which is necessary for the synthesis of fatty acids and cholesterol and thus represents an important molecular target for lowering lipids (Hatzivassiliou et al., 2005; Xie et al., 2015). ACLY is abnormally expressed in many cancers, CVDs, and metabolic disorders (Bazilevsky et al., 2019). A previous study showed that ACLY expression in macrophages increased during treatment with proinflammatory stimuli, lipopolysaccharide (LPS), tumor necrosis factor $\alpha(\mathrm{TNF} \alpha)$, and Interferon gamma (IFN $\gamma$ ) (Infantino et al., 2013). Interestingly, the pharmacological inhibition of ACLY by SB-204990 attenuated the inflammatory response and oxidative stress in activated macrophages obtained from mouse bone marrow (Infantino et al., 2013).

In addition, the IL-4 M2 polarizing agent increases ACLY phosphorylation and AcCoA production in M2 macrophages via the Akt-dependent pathway (Covarrubias et al., 2016). However, studies conducted on macrophages originating from human monocytes suggest that ACLY was not necessary for IL-4-induced M2 polarization using human macrophages in which ACLY was reduced or knocked out (Namgaladze et al., 2018). These conflicting results can be caused by the different types of cells used and the inappropriate effects of ACLY inhibitors. These recent studies have shown that the exact role of ACLY in macrophage activation and polarization remains to be confirmed in vitro and in vivo. There is currently no information on whether ACLY deletion or pharmacological inhibition affects the biosynthesis of TG and cholesterol in macrophages.

Moreover, liver-specific ACLY deletion prevents liver steatosis, while fat-specific ACLY deficiency does not have a phenotype (Zhao et al., 2016). A possible reason may be that ACLY breaks down citrate to form AcCoA outside mitochondria for de novo glucose-dependent lipogenesis, and ACLY expression is strongly regulated by the presence of nutrients in adipocytes, and its expression is induced by carbohydrates and suppressed by dietary fiber. Thus, the presence or absence of ACLY in adipocytes should not have any significant effect on lipid metabolism, unless the mice are on a high carbohydrate diet. It has recently been reported that the sexual dimorphic function of ACLY derived from adipocytes supports systemic metabolic homeostasis by activating the nutrient-dependent carbohydrate response elementbinding protein (Fernandez et al., 2019). These data indicate that the presence of the phenotype in the absence of ACLY in adipose tissue depends on gender and diet, thus adding complexity to the functions of ACLY in the regulation of cardiometabolic disorders.

The sterol transporter ATP-binding cassette transporter G5/8 (ABCG5/8) regulates the final stage of reverse cholesterol transport, which promotes hepatobiliary transport of cholesterol. ACLY inhibition has an antiatherosclerotic effect due to increased expression of ABCG5/8 (Molusky et al., 2018). The depletion of AcCoA levels induces an autophagic flow, while increased levels of cytosolic AcCoA effectively inhibit autophagy. Dimethyl $\alpha$-ketoglutarate (DMKG) increases intracellular levels of $\alpha$-ketoglutarate, which is converted to AcCoA by isocitrate dehydrogenase (IDH1 or IDH2) and ACLY. Repeated treatment with DMKG inhibits myocardial autophagy in mice undergoing thoracic aortic narrowing, while eliminating pathological heart remodeling (Marino et al., 2014). In addition, carboxylesterase 1 (CES1) is an important enzyme that hydrolyzes TG and cholesterol esters. Knockdown of CES1 in mouse liver significantly increases blood glucose levels after meals. ACLY can also regulate histone acetylation, while ACLY knockout inhibits glucose-induced histone acetylation of CES1 and CES1 expression in the liver (Xu et al., 2014).

BemA is a new drug that treats dyslipidemia and other CVDs. It has been identified from a number of long hydrocarbon chains that inhibit the synthesis of cholesterol and fatty acids in vitro and in vivo (Oniciu et al., 2006; Pinkosky et al., 2013). BemA rapidly forms BemA-CoA in the liver (thus, functioning as a direct and potent competitive ACLY inhibitor), which reduces the levels of non-HDL cholesterol, and TG and insulin and also increases the plasma $\beta$-hydroxybutyrate level in obese Zucker (fa/ fa) rat (Pinkosky et al., 2013).

Mounting studies have shown that BemA has pharmacological effects, especially in the liver (Pinkosky et al., 2013, 2016). A very long chain acyl-CoA synthetase-1 (ACSVL1) is required to convert BemA into its active coenzyme A derivative. ACSVL1 is an enzyme specifically expressed in the liver that provides the theoretical basis for preventing potential muscle side effects. In addition to ACLY inhibition, BemA can also activate the fuel sensor AMP-activated protein kinase (AMPK) in rodents, although the effect of AMPK activation is not observed in humans. In particular, BemA selectively activates AMPK $\beta 1$ in mice instead of $\beta 2$ (Pinkosky et al., 2016).

\section{Effect of bempedoic acid on lipid metabolism}

BemA has a mechanism of action similar to that of statins, which inhibits ACLY-dependent cholesterol biosynthesis, a step prior to $\beta$-Hydroxy $\beta$-methylglutaryl-CoA (HMG-CoA) reductase. Since BemA is a prodrug that is activated only in the liver, therefore, BemA has minimal myotoxicity (unlike statins) (Katwan et al., 2019). In patients with statin intolerance who need to achieve a significant reduction in the risk of CVD, the high tolerance of BemA makes it a useful alternative, either alone or in combination with EZE. BemA treatment is also associated with a significant reduction in the biomarker of systemic inflammation, such as hsCRP (Goldberg et al., 2019).

In five randomized controlled trials (RCTs) that were systematically evaluated, BemA was better than placebo in lowering serum levels of LDL cholesterol, non-HDL cholesterol, and apoB. Compared to EZE, BemA is superior in lowering LDL cholesterol. From a safety point of view, the frequency of all adverse events (including headache, joint pain, and myalgia) did not statistically differ between the BemA groups and placebo, suggesting that BemA seems to be an appropriate drug in patients with statin intolerance (Wang et al., 2019).

BemA requires activation by the specific enzyme ACSVL1, which is largely limited to the liver. Therefore, it is believed that unlike statins, myotoxicity is unlikely to occur with BemA, since it does not inhibit CSVL1 in these cells (Pinkosky et al., 2016). The BemA effect is additive rather than redundant compared to statins, because the BemA target (ACLY) is an excellent regulatory reference point for cholesterol biosynthesis 
than HMG-CoA reductase, the main target of statins. Failure to tolerate statins due to muscle symptoms contributes to uncontrolled cholesterol levels and an insufficient reduction in cardiovascular risk. The mechanism of action of BemA is similar to the mechanism of action of statins, but since it does not inhibit the pathway of cholesterol biosynthesis in skeletal muscle, thereby providing a mechanistic basis for reducing the potential for side effects associated with muscle (Filippov et al., 2013).

Animal experiments and clinical trials in humans have shown that ACLY inhibitors can significantly improve dyslipidemia (especially lowering LDL cholesterol) and inhibit atherosclerotic lesions (Burke et al., 2017; Ruscica et al., 2019). These inhibitors have a comparable effect with statins and have some potential advantage over other nonstatin hypolipidemic drugs (in lowering the level of LDL cholesterol). Among these inhibitors, BemA is the only liver-specific ACLY inhibitor that has been approved for clinical trials, and it is a prodrug that has a lipidlowering effect by ACLY inhibition, which requires activation by ACSVL1. Therefore, BemA can serve as a good alternative and additional medicine for patients who cannot use high doses of statins due to side effects.

In people with dyslipidemia, BemA not only lowered LDL cholesterol in the blood but also significantly reduced the level of hsCRP, a clinical biomarker of systemic inflammation. BemA also inhibits inflammatory responses in primary macrophages derived from human monocytes and in mice. Fundamental mechanisms include the signaling pathways of hepatic kinase B1 serine-threonine kinase 11 (LKB1)/AMPK, mitogen-activated protein kinase, and c-Jun N-terminal kinase (Filippov et al., 2013).

Individual differences in drug effects have always been an important part of clinical pharmaceutical research and precision medicine (Ahmad et al., 2011; Guo et al., 2018). However, there is still no clear study of the relationship between the genetic variants of ACSVL1 and its enzymatic activity. In addition, BemA targets are upstream of statins, while ApoE-E4-carrying patients undergoing statin therapy are less effective (Thompson et al., 2009). Therefore, the ApoE genotype can also influence the effectiveness of BemA.

In addition, during remodeling of the heart caused by acute myocardial infarction, ACLY expression was significantly increased (Guo et al., 2018), suggesting that ACLY may play an important role in heart hypertrophy and heart failure. All these data confirm the prospect of ACLY inhibition for reducing CVDs and other metabolic disorders (Pinkosky et al., 2017). However, further studies are needed in the future to clarify the exact role of ACLY in heart disease and to evaluate the side effects of BemA, not associated with myopathy, in large-scale, randomized clinical trials in patients with CAD.

\section{Effect of bempedoic acid on atherosclerosis in preclinical models}

Studies in LDLR-/- minipigs and mice, and ApoE-/mice have shown that BemA lowers LDL cholesterol and inhibits atherosclerosis. In LDLR $+/-$ and LDLR-/- pigs fed a high fat diet, BemA was administered to pigs for 160 days. In LDLR+/pig aorta, BemA reduced total cholesterol and LDL cholesterol by $40 \%$ and $61 \%$, respectively. At the same time, BemA significantly reduced the en face area $(-58 \%)$ and the lesion area in the left anterior descending coronary artery (-40\%). In LDLR -/- pigs, BemA reduced plasma cholesterol and LDL cholesterol by $27 \%$ and $29 \%$, respectively. Moreover, BemA reduced the area of aortic lesion $(-47 \%)$ and the left-sided lesion of the descending coronary artery $(-48 \%)$ (Burke et al., 2019). In LDLR-/- mice fed a diet high in fat and cholesterol, treatment with BemA for 12 weeks (at doses of 3,10 , and $30 \mathrm{mg} / \mathrm{kg} /$ day) leads to a decrease in lipid accumulation in plasma, liver, and aorta, since as well as weakened aortic inflammation (attenuates the proinflammatory expression of the M1 gene). BemA dose-dependently reduces hypertriglyceridemia, hyperinsulinemia, hypercholesterolemia, hyperglycemia, aortic sclerosing lesions, , and obesity (Samsoondar et al., 2017).

In ApoE-/- and double-knockout (DKO) (ApoE-/- and Ampk $\beta 1-/-$ ) mice, BemA (30 mg/kg/day, 12 weeks) significantly inhibited atherosclerotic lesions of the aorta. In addition, BemA treatment reduced TG and LDL cholesterol levels in both genotype mice, while plasma amyloid A serum amyloid A (SAA) levels also decreased significantly (Pinkosky et al., 2016). These results show that the antiatherosclerotic effect of BemA mainly regulates lipid metabolism in the liver and reduces LDL cholesterol levels and inflammation, regardless of AMPK activation. The results of the above-mentioned animal experiments convincingly demonstrate the protective effect of BemA in atherosclerosis, and this mechanism mainly involves the regulation of lipid metabolism and the inhibition of inflammation.

\section{Clinical efficacy of bempedoic acid}

BemA has wide clinical efficacy in patients with CVD diseases as monotherapy or combination therapy with other lipid modulating drugs.

\section{Phase 1 studies}

A first-in-human, phase 1a single-dose clinical trial, ETC-1002-001 (Esperion Therapeutics, 2015) evaluated safety, tolerability, and pharmacokinetics of ETC-1002 in 18 healthy subjects. Similarly, ETC-1002-002 (ClinicalTrials.gov) was a staged phase $1 \mathrm{~b}$ multiple-dose tolerance clinical trial over 2 and 4 weeks in 53 patients, 39 of whom received ETC-1002 and 23 received placebo. Subjects were divided into four different cohorts of six subjects, each of whom received 20,60,100, or $120 \mathrm{mg}$ of ETC-1002 or placebo once a day for 14 days. This was followed by the study of a larger cohort, which was treated for 28 days, during which the subjects lived outside the clinical site for the entire treatment period. ETC-1002 was safe, well tolerated, and associated with no dose-limiting side effects.

Finally, ETC-1002-004 (ClinicalTrials.gov) was a 2-week, phase $1 \mathrm{~b}$, clinical trial of multiple-dose tolerance in 24 subjects, 18 of which received ETC-1002. This clinical trial was designed to evaluate the safety and tolerability of increasing multiple oral doses of ETC-1002 above $120 \mathrm{mg} /$ day. Subjects in this clinical trial received 140,180 , or $220 \mathrm{mg}$ of ETC-1002 or placebo once a day for 14 days. LDL cholesterol levels were reduced by an average of $36 \%$ for subjects receiving $220 \mathrm{mg} /$ day of ETC-1002, compared with an increase of 4\% for subjects receiving placebo $(p<0.0001)$. Subjects receiving ETC-1002 showed no serious side effects. ETC-1002 was safe, well tolerated, and associated with no dose-limiting side effects. 
Phases 2 and 3 studies

\section{Bempedoic acid monotherapy}

In a phase 2 clinical trial, 60 patients with hyperlipidemia and type 2 diabetes mellitus who discontinued antidiabetic and lipid modulating drugs were randomly assigned to the BemA group (80 mg of ETC-1002 once daily for 2 weeks, followed by 120 mg of ETC-1002 once daily for 2 additional weeks) and placebo (Gutierrez et al., 2014). LDL cholesterol levels after 4 weeks of treatment with ETC-1002, which was the primary endpoint, were reduced by an average of $43 \%$ in patients receiving the $120 \mathrm{mg}$ ETC-1002 dose, compared with an average of $4 \%$ in patients receiving placebo $(p<0.0001)$. Approximately, $80 \%$ of the patients were not at their National Cholesterol Education Program Adult Treatment Panel III LDL-C goal of less than $100 \mathrm{mg} / \mathrm{dl}$ at the beginning of the study. Of these, $88 \%$ of patients receiving ETC-1002 achieved their goal by the end of the study compared to $4 \%$ of patients receiving placebo $(p<0.0001)$. HsCRP levels were reduced by $41 \%$ at the dose of ETC- 1002 of $120 \mathrm{mg}$ compared to $11 \%$ with placebo $(p=0.001)$. Non-HDL cholesterol decreased by $32 \%$ in patients receiving ETC-1002, compared with a $1 \%$ increase in patients receiving placebo $(p<0.0001)$. A 24-hour continuous glucose assessment showed a slight improvement in glycemic control in the treatment of ETC-1002. Overall, 24-hour ambulatory blood pressure monitoring did not show differences between treatment groups in mean changes from baseline to day 28.

In another phase II clinical trial, 177 patients with elevated LDL cholesterol were randomized to receive BemA (40, 80 , or $120 \mathrm{mg}$ once daily for 12 weeks) or placebo (Ballantyne et al., 2013). This clinical study was designed to evaluate the efficacy and safety of LDL lowering with ETC-1002 compared with placebo in patients with hypercholesterolemia (LDL from 130 to $220 \mathrm{mg} / \mathrm{dl}$ ) and normal TG (less than $150 \mathrm{mg} / \mathrm{dl}$ ) or elevated TG (150-400 mg/dl). The four groups were placebo and 40, 80, and $120 \mathrm{mg}$ doses of ETC-1002 once daily. LDL cholesterol levels were reduced on average by $18 \%, 25 \%$, and $27 \%$ for patients receiving ETC-1002, 40, 80, and $120 \mathrm{mg}$, respectively, compared with an average of $2 \%$ for patients receiving placebo $(p<0.0001)$. ETC-1002's lowering of LDL-C levels was maintained across a range of baseline triglyceride levels. ETC-1002 also reduced levels of atherogenic biomarkers apo B, non-HDL cholesterol, and LDL particle number $(p<0.0001)$ in a dose-dependent manner. Patients treated with ETC-1002 showed a tendency to decrease hsCRP from $20 \%$ to $26 \%$ compared with $2 \%$ in patients treated with placebo. In the subgroup of patients with increased hsCRP, patients receiving ETC-1002 showed a tendency to decrease hsCRP from $43 \%$ to $64 \%$ compared with a decrease of $7 \%$ in patients receiving placebo.

In a 6-week, multicenter, randomized, double-blind, placebo-controlled study in a parallel group phase 2 , the safety and efficacy of ETC-1002 were compared with placebo in 143 patients with both hypercholesterolemia and hypertension (ClinicalTrials. gov). After washout of any lipid-modifying therapy and blood pressure therapy, 71 patients received $180 \mathrm{mg}$ ETC-1002 and 72 patients received placebo. Patients treated with ETC-1002 achieved a $21 \%$ reduction in LDL cholesterol after 6 weeks compared with a $3 \%$ increase in LDL cholesterol in the placebo group $(p<0.0001)$. The decrease occurred during the first 2 weeks after the start of therapy and continued throughout the treatment period. HsCRP levels were reduced by $25 \%$ with ETC-1002, compared with a $20 \%$ increase in the placebo group $(p<0.0001)$. ETC-1002 had a neutral effect on blood pressure and was safe and well tolerated. Despite the effective reduction in LDL cholesterol using ETC1002, HDL cholesterol and triglyceride levels were unchanged in all treatment groups in these studies. In patients receiving ETC1002, no serious side effects were observed. ETC-1002 was safe, well tolerated, and associated with no dose-limiting side effects.

\section{Bempedoic acid add-on to statin therapy}

In a phase 2 clinical trial, 134 patients with hyperlipidemia undergoing statin treatment were randomized to receive additional treatment with BemA 120, $180 \mathrm{mg}$, or placebo for 12 weeks. It was observed that 120 or $180 \mathrm{mg}$ of BemA dose-dependently reduced the level of LDL cholesterol, apoB, non-HDL cholesterol, total cholesterol, and LDL particles compared to placebo. The incidence of muscle side effects and treatment interruption was the same in both groups (Ballantyne et al., 2016).

In an 8-week, phase 2 a clinical trial in 58 patients, of which 42 were treated with ETC-1002, the primary endpoint was the number of patients with side effects, clinical laboratory abnormalities, and other safety data (ClinicalTrials.gov). ETC1002 as a supplement to $10 \mathrm{mg}$ of atorvastatin was well tolerated and did not lead to any serious side effects. Although the study was not designed to evaluate the decrease in LDL cholesterol using ETC-1002, it was measured as a secondary endpoint to determine if a decrease in LDL-C would be observed with the addition of ETC-1002 during statin therapy. In patients treated with atorvastatin, ETC-1002 lowered LDL-C levels by an average of $22 \%$ compared with a $0 \%$ change in placebo $(p<0.0001)$.

The following ETC-1002 study (Ballantyne et al., 2015) was a double-blind, placebo-controlled, multicenter, parallelgroup study that evaluated 134 patients with baseline LDL cholesterol levels of $115-220 \mathrm{mg} / \mathrm{l}$, while taking atorvastatin $\leq 20$ $\mathrm{mg}$, simvastatin $\leq 20 \mathrm{mg}$, rosuvastatin $\leq 10 \mathrm{mg}$ or pravastatin $\leq 40$ $\mathrm{mg}$ randomized to receive $120 \mathrm{mg}$ of ETC-1002, $180 \mathrm{mg}$ of ETC1002 , or placebo once a day for 12 weeks. ETC-1002 lowered LDL levels by up to $24 \%(p<0.0001)$, significantly more than placebo, in addition to statin therapy. ETC-1002 also lowered ( $p$ $<0.05$ ) levels of apo B and non-HDL cholesterol, as well as total cholesterol and LDL particle number by more than placebo. A slight decrease in hsCRP was observe with ETC-1002, $120 \mathrm{mg}$ $(22 \%, p=0.26)$, and $180 \mathrm{mg}(30 \%, p=0.08)$ compared to $0 \%$ with placebo. No significant changes in HDL cholesterol or TG levels were observed in any study. Adverse event associated with muscle events, discontinuation of treatment due to adverse events, and clinical safety laboratory levels were generally similar compared to placebo .

In addition, 68 patients with hypercholesterolemia who received atorvastatin $80 \mathrm{mg}$ for 4 weeks were randomly divided into an experimental group (BemA $180 \mathrm{mg}+$ atorvastatin 80 $\mathrm{mg}$ ) and a control group (placebo + atorvastatin $80 \mathrm{mg}$ ) in a 2:1 
ratio (phase 2). After 4 weeks, the level of LDL cholesterol, total cholesterol, apoB, and hsCRP in the experimental group was significantly lower than that in the control group. In addition, by determining the level of atorvastatin and its metabolites, it was found that BemA $180 \mathrm{mg}$ does not increase the clinical exposure of atorvastatin (Lalwani et al., 2019). These studies show that BemA can further improve blood lipids in hyperlipidemic patients taking large doses of statins (Table 2).

In addition, a recently published randomized controlled clinical trial involved 2,230 patients (with CVD, with/without heterozygous familial hypercholesterolemia) who received the most tolerated dose of statin therapy. Of these patients, 1,488 were prescribed for BemA (180 mg once daily, 52 weeks) and 742 were prescribed for placebo. The results showed that the level of LDL cholesterol in the BemA group was significantly lower, with no apparent difference in the frequency of adverse events between the two groups (Ray et al., 2019).

\section{Bempedoic acid in statin-intolerant patients}

Fifty-six patients with statin intolerance were randomized to receive BemA or placebo in a 2:1 ratio (phase 2). The initial dose of BemA was $60 \mathrm{mg}$ per day, which was increased to 120,180 , and $240 \mathrm{mg}$ at 2 -week intervals for a total of 8 weeks. The results showed that BemA treatment reduced LDL-C by $28.7 \%$ compared with placebo. Moreover, BemA significantly reduced levels of non-HDL cholesterol, apoB, total cholesterol, and hsCRP. However, in the BemA group, there were no significant differences in the levels of TG and HDL cholesterol. The frequency of side effects associated with muscle was the same in both groups (Thompson et al., 2015).

In a phase 2 clinical trial, 349 hypercholesterolemic patients with or without statin intolerance were randomly administered BemA 120, 180 mg, EZE, BemA 120 mg + EZE, or BemA $180 \mathrm{mg}+\mathrm{EZE}$ for 12 weeks. With a decrease in LDL cholesterol, EZE, BemA (120 and $180 \mathrm{mg})$, and BemA (120 mg + EZE and $180 \mathrm{mg}+\mathrm{EZE}$ ) were reduced by $21 \%, 27 \%, 30 \%, 43 \%$, and $48 \%$, respectively. Compared to EZE alone, using BemA alone or in combination with EZE also reduces non-HDL cholesterol, total cholesterol, apoB, LDL, and hsCRP particles. BemA was safe, effective, and well tolerated, and the incidence of muscle side effects was the same in all treatment groups (Thompson et al., 2016).

In phase 3 of the clinical trial, 345 patients with hypercholesterolemia not tolerating at least two statins (one of the statins was intolerant at the lowest available dose) were randomly administered to the BemA group (180 mg, one time per day) or placebo group at 2:1. The results showed that BemA treatment significantly reduced LDL cholesterol (-21.4\%), non-HDL cholesterol $(-17.9 \%)$, total cholesterol $(-14.8 \%)$, apoB $(-15$, $0 \%$ ), and hsCRP (-24.3\%). The incidence of muscle side effects in the BemA and placebo groups was $4.7 \%$ and $7.2 \%$, respectively. These results indicate that, in patients with statin intolerance, BemA provides a safe and effective lipid-lowering effect (Laufs et al., 2019).

Another phase 3 clinical study included 269 patients with statin intolerance. After 4 weeks of EZE treatment (10 $\mathrm{mg} /$ day), the patients were randomly assigned (2:1 ratio) to the BemA $180 \mathrm{mg}+$ EZE $10 \mathrm{mg}$ group and the EZE $10 \mathrm{mg}+$ placebo group for 12 weeks. The results showed that, compared with placebo, BemA lowered LDL cholesterol by $28.5 \%$, total cholesterol by $18.0 \%$, non-HDL cholesterol by $23.6 \%$, apoB by $19.3 \%$, and hsCRP by $31.0 \%$ [76]. In addition, the incidence of muscle-related adverse events and interruptions was the same in both groups. These results suggest that BemA may be an additional treatment option for patients with statin intolerance, but requires a significant reduction in LDL cholesterol (Ballantyne et al., 2018).

\section{Phase 3 program CLEAR (cholesterol lowering via bempedoic acid, an ACLY-inhibiting regimen)}

The phase 3 CLEAR Serenity clinical trial demonstrated the lipid-lowering efficacy of BemA among patients with established statin intolerance and elevated LDL cholesterol who received stable background therapy. Although BemA acts on the same cholesterol biosynthesis pathway as statins, the incidence of muscle-related side effects in CLEAR Serenity with BemA is no different from placebo, even among patients who have symptoms, associated with the muscles, while being on statin therapy (Banach et al., 2019; Ray et al., 2019).

In the CLEAR Harmony study (Evaluation of LongTerm Safety and Tolerability of ETC-1002 in High-Risk Patients with Hyperlipidemia and High Cardiovascular Risk), patients who received maximally tolerated therapy with statins treated with BemA had significantly lower LDL cholesterol than which received a placebo (mean difference, 18\%), without an increase in serious side effects (Ray et al., 2019).

The efficacy and safety of BemA will be further defined in a larger study of cardiovascular outcomes, CLEAR Outcome [Evaluation of Major Cardiovascular Events in Patients with, or at High Risk for, CVD Who Are Statin Intolerant Treated with Bempedoic Acid (ETC-1002) or Placebo], which includes 12,600 patients with high cardiovascular risk who have side effects in response to statins and have LDL cholesterol level of $100 \mathrm{mg}$ per deciliter or higher (Banach et al., 2019).

In the published systematic review with meta-analysis of RCTs to assess safety and efficacy of $180 \mathrm{mg}$ BemA in patients with hypercholesterolemia, it has been shown that a more significant reduction in LDL cholesterol (MD, $-17.5 \%$; 95\% CI, $-22.9 \%$ to $-12.0 \%$ ), total cholesterol (MD, $-10.9 \%$; $95 \% \mathrm{CI}$, $-13.3 \%$ to $-8.5 \%$ ), non-HDL cholesterol (MD, $-12.3 \%$; $95 \% \mathrm{CI}$, $-15.3 \%$ to $-9.20 \%$ ), apo B (MD, $-10.6 \% ; 95 \% \mathrm{CI},-13.2 \%$ to $-8.02 \%$ ), and hsCRP (MD, $-13.2 \% ; 95 \% \mathrm{CI},-16.7 \%$ to $-9.79 \%)$ in BA-treated patients compared with controls. Results were confirmed when separately analyzing studies on patients with high cardiovascular risk, studies on statin-intolerant patients, and studies on patients with hypercholesterolemia on maximally tolerated lipid-lowering therapy. BemA-treated subjects reported a higher rate of treatment discontinuation caused by adverse effects, of gout flare, and of increase in uric acid compared with controls. On the other hand, BA-treated patients showed a lower incidence of new-onset diabetes mellitus than controls (Di Minno et al., 2020). 
Table 2. Bempedoic acid.

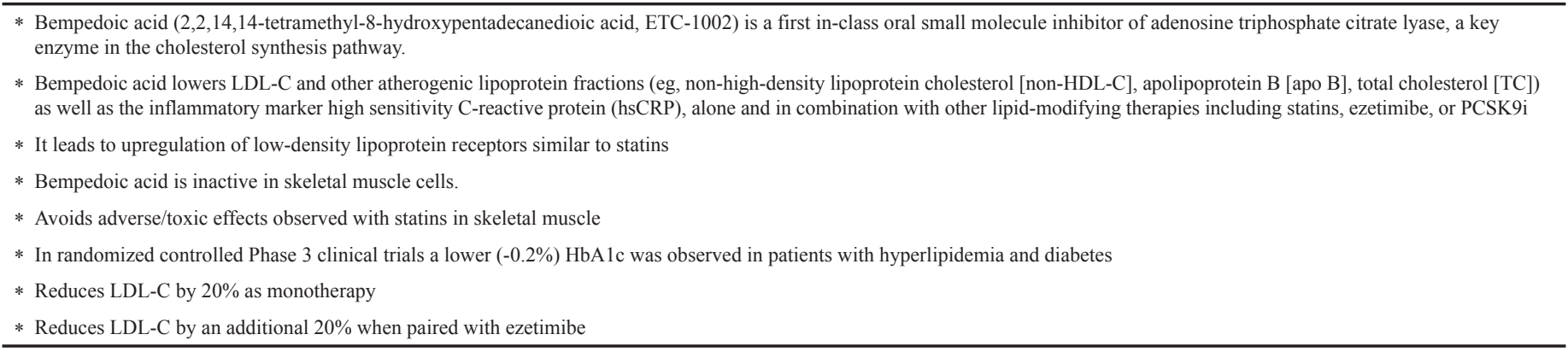

\section{Conclusion}

The current clinical trial results suggest that BemA(ETC1002) may represent a new therapeutic approach for lowering LDL cholesterol. BemA is a prodrug specifically activated in the liver, where it inhibits ACLY, a regulatory checkpoint within the cholesterol biosynthesis pathway. By inhibiting ACLY, BemA reduces the synthesis of cholesterol in the liver cells and triggers the compensatory activation of the LDL receptor, as well as complementing other mechanisms targeted by current therapies, which leads to an additional decrease in LDL cho lesterol, without leading to increases in adverse events.

BemA for lowering lipid levels is showing promising results in early clinical trials and is awaiting confirmation of benefit and safety in large cardiovascular outcome studies. The Food and Drug Administration (FDA) has approved BemA for the treatment of adults with heterozygous familial hypercholesterolemia or established atherosclerotic CVD who require additional lowering of LDL cholesterol. The 2019 European Society of Cardiology/ European Atherosclerosis Society guidelines for the management of dyslipidemias recommend BemA as a potential novel therapy for lowering LDL cholesterol.

The potential value for cardiovascular risk reduction will depend on the results of the pending cardiovascular outcomes trial. Long-term safety data and effect on cardiovascular outcomes are needed. The results of these studies may change future lipid management practices.

\section{CONFLICT OF INTEREST}

All the authors declare that they have no conflicts of interest for this work.

\section{FUNDING}

None.

\section{REFERENCES}

Ahmad T, Voora D, Becker RC. The pharmacogenet of antiplatelet agents: towards personalized therapy? Nat Rev Cardiol, 2011; 8(10):560-71.

Arnett DK, Blumenthal RS, Albert MA, Buroker AB, Goldberger ZD, Hahn EJ, Himmelfarb CD, Khera A, Lloyd-Jones D, McEvoy JW, Michos ED, Miedema MD, Muñoz D, Smith Jr SC, Virani SS, Williams Sr KA, Yeboah J, Ziaeian B. 2019 ACC/AHA guideline on the primary prevention of cardiovascular disease: executive summary: a report of the American College of Cardiology/American Heart Association Task Force on Clinical Practice Guidelines. J Am Coll Cardiol, 2019; 74:1376-414.

Assessment of the long-term safety and efficacy of bempedoic acid (CLEAR Harmony OLE) (2019). https://clinica.gov/ct2/show/ NCT0306744.
Ballantyne CM, Banach M, Mancini GBJ, Lepor NE, Hanselman JC, Zhao X, Leiter LA. Efficacy and safety of bempedoic acid added to ezetimibe in statin-intolerant patients with hypercholesterolemia: a randomized, placebo-controlled study. Atherosclerosis, 2018; 277: 195-203.

Ballantyne CM, Davidson MH, Macdougall DE, Bays HE, Dicarlo LA, Rosenberg NL, Margulies J, Newton RS. Efficacy and safety of a novel dual modulator of adenosine triphosphate-citrate lyase and adenosine monophosphate-activated protein kinase in patients with hypercholesterolemia: results of a multicenter, randomized, doubleblind, placebo-controlled, parallel-group trial. J Am Coll Cardiol, 2013; 62(13):1154-62.

Ballantyne CM, Macdougall DE, Margulies JR, Robinson PL, Hanselman JC, Lalwani ND. ETC-1002 incrementally lowers low density lipoprotein-cholesterol in patients with hypercholesterolemia receiving stable statin therapy [abstract 17499]. Circulation, 2015; 132:A17499.

Ballantyne CM, McKenney JM, MacDougall DE, Margulies JR, Robinson PL, Hanselman JC, Lalwani ND. Effect of ETC-1002 on serum low-density lipoprotein cholesterol in hypercholesterolemic patients receiving statin therapy. Am J Cardiol, 2016; 117(12):1928-33.

Banach M, Duell BP, Gotto A, Laufs U, Leiter LA, Mancini GBJ, Ray KK, Flaim J, Ye Z, Catapano AL. Efficacy of bempedoic acid: a pooled analysis of 4 pivotal Phase III clinical trials. Circulation, 2019; 140:A12268.

Bazilevsky GA, Affronti HC, Wei X, Campbell SL, Wellen KE, Marmorstein R. ATP-citrate lyase multimerization is required for coenzyme-a substrate binding and catalysis. J Biol Chem, 2019; 294(18):7259-68

Bilen O, Ballantyne CM. Bempedoic acid (ETC-1002): an investigational inhibitor of ATP citrate lyase. Curr Atheroscler Rep, 2016; 18(10):61.

Bonaca MP, Nault P, Giugliano RP, Keech AC, Pineda AL, Kanevsky E, Kuder J, Murphy SA, Jukema JW, Lewis BS, Tokgozoglu L, Somaratne R, Sever PS, Pedersen TR, Sabatine MS. Low-density lipoprotein cholesterol lowering with evolocumab and outcomes in patients with peripheral artery disease: insights from the FOURIER trial (Further Cardiovascular Outcomes Research With PCSK9 Inhibition in Subjects With Elevat- ed Risk). Circulation. 2018; 137:338-50.

Brinton EA. Management of hypertriglyceridemia for prevention of atherosclerotic cardiovascular disease. Cardiol Clin, 2015; 33: 309-23.

Burke AC, Huff MW. ATP-citrate lyase: genetics, molecular biology and ther- apeutic target for dyslipidemia. Curr Opin Lipidol, 2017; 28(2):193-200.

Burke AC, Telford DE, Huff MW. Bempedoic acid: effects on lipoprotein metabolism and atherosclerosis. Curr Opin Lipidol, 2019; 30(1):1-9.

ClinicalTrials.gov. A multiple ascending dose study of ETC1002 in healthy subjects. Available via: https://clinicaltrials.gov/ct2 /show/ NCT01485146, 2011

ClinicalTrials.gov. A multiple ascending dose study of ETC1002 in subjects with mild dyslipidemia. Available via: https://clinicaltrials. gov/ct2/show/NCT01105598, 2010 
Covarrubias AJ, Aksoylar HI, Yu J, Snyder NW, Worth AJ, Iyer SS, Wang J, Ben-Sahra I, Byles V, Polynne-Stapornkul T, Espinosa EC, Lamming D, Manning BD, Zhang Y, Blair IA, Horng T. Akt-mTORC1 signaling regulates ACLY to integrate metabolic input to control of macrophage activation. eLife, 2016; 5:e11612.

Di Minno A, Lupoli R, Calcaterra I, Poggio P, Forte F, Spadarella G, Ambrosino P, Iannuzzo G, Di Minno MND. Efficacy and safety of bempedoic acid in patients with hypercholesterolemia: systematic review and meta-analysis of randomized controlled trials. J Am Heart Assoc, 2020; 9:e016262; doi: 10.1161/JAHA.119.016262.

Esperion announces positive CHMP opinion for the marketing authorisation application for bempedoic acid for the treatment of hypercholesterolemia and mixed dyslipidemia. 2020. Available via https:// www.esperion.com/investors-media/news-releases/news-release-details/ esperion-announces-us-fda-acceptance-new-drug-applications-ndas/

Esperion announces positive CHMP opinion for the marketing authorisation application for the bempedoic acid/ezetimibe fixed dose combination tablet for the treatment of hypercholesterolemia and mixed dyslipidemia. 2020. Available via https://www.esperion.com/investorsmed ia/news-releases/news-release-details/esperion-announces-us-fdaacceptance-new-drug-applications-ndas/

Esperion Announces U.S. FDA acceptance of new drug applications (NDAs) for both bempedoic acid and the bempedoic acid/ ezetimibe combination tablet for filing and regulatory review. 2019. Available via https://www.esperion.com/investors-media/press releases/

Esperion Therapeutics I. Form 10-K - Annual report United States securities and exchange commission. 2015. Available via http://www. sec.gov/Archives/edgar/data/1434868/000104746915001908/000104746915-001908-index.htm.

Evaluation of ETC-1002 in patients with hypercholesterolemia and hypertension. 2019. Available via https://clinicaltrials.gov/ct2/show/ NCT02178098

Evaluation of the efficacy and safety of bempedoic acid (ETC1002) $180 \mathrm{mg}$ when added to PCSK9 inhibitor therapy. 2019. https:// clinicaltrials.gov/ct2/show/NCT03193047

Evaluation of the efficacy and safety of bempedoic acid (ETC1002) $180 \mathrm{mg}$, ezetimibe $10 \mathrm{mg}$, and atorvastatin $20 \mathrm{mg}$ triplet therapy in patients with elevated LDL-C. 2019. Available via https://clinicaltrials.gov/ ct2/show/NCT03051100

Evaluation of the efficacy and safety of bempedoic acid (ETC1002) in patients with hyperlipidemia and statin intolerant (CLEAR Serenity). 2019. Available via https://clinicaltrials.gov/ct2/show/ NCT02988115

Ference BA, Ray KK, Catapano AL, Ference TB, Burgess S, Neff DR, Oliver-Williams C, Wood AM, Butterworth AS, Angelantonio ED, Danesh J, Kastelein JJP, Nicholls SJ. Mendelian randomization study of ACLY and cardiovascular disease. N Engl J Med, 2019; 380(11):1033-42.

Fernandez S, Viola JM, Torres A, Wallace M, Trefely S, Zhao S, Affronti HC, Gengatharan JM , Guertin DA, Snyder NW, Metallo CM, Wellen KE. Adipocyte ACLY facilitates dietary carbohydrate handling to maintain metabolic homeostasis in females. Cell Rep. 2019; 27(9):2772-84.

Filippov S. Pinkosky SL, Lister RJ, Pawloski C, Hanselman JC, Cramer CT, Srivastava RAK, Hurley TR, Bradshaw CD, Spahr MA, Newton RS. ETC-1002 regulates immune response, leukocyte homing, and adipose tissue inflammation via LKB1-dependent activation of macrophage AMPK. J Lipid Res, 2013; 54(8):2095-108.

Goldberg AC, Leiter LA, Stroes ESG, Baum SJ, Hanselman JC, Bloedon LT, Lalwani ND, Patel PM, Zhao X, Duell PB. Effect of bempedoic acid vs placebo added to maximally tolerated statins on low-density lipoprotein cholesterol in patients at high risk for cardiovascular disease the clear wisdom randomized clinical trial. JAMA, 2019; 322(18):1780-8.

Grundy SM, Stone NJ, Bailey AL, Beam C, Birtcher KK, Blumenthal RS, Braun LT, de Ferranti S, Faiella-Tommasino J, Forman DE, Goldberg R, Heidenreich PA, Hlatky MA, Jones DW, LloydJones D, Lopez-Pajares N, Ndumele CE, Orringer CE, Peralta CA,
Saseen JJ, Smith Jr SC, Sperling L, Virani SS, Yeboah J. 2018 AHA/ ACC/AACVPR/AAPA/ABC/ACPM/ADA/AGS/APhA/ASPC/NLA/ PCNA guideline on the management of blood cholesterol: executive summary: a report of the American College of Cardiology/American Heart Association Task Force on Clinical Practice Guidelines. J Am Coll Cardiol, 2019; 73:3168-209.

Guo N, Zhang N, Yan L, Lian Z, Wang J, Lv F, Wang Y, Cao X. Weighted gene coexpression network analysis in identification of key genes and networks for ischemic reperfusion remodeling myocardium. Mol Med Rep, 2018; 18(2):1955-62.

Gutierrez MJ, Rosenberg NL, Macdougall DE. Efficacy and safety of ETC-1002, a novel investigational low-density lipoproteincholesterol-lowering therapy for the treatment of patients with hypercholesterolemia and type 2 diabetes mellitus. Arterioscler Thromb Vasc Biol, 2014; 34(3):676-83.

Hatzivassiliou G, Zhao F, Bauer DE, Andreadis C, Shaw AN, Dhanak D, Hingorani SR, Tuveson DA, Thompson CB. ATP citrate lyase inhibition can suppress tumor cell growth. Cancer Cell, 2005; 8(4):311-21. Infantino V, Iacobazzi V, Palmieri F, Menga A. ATP-citrate lyase is essential for macrophage inflammatory response. Biochem Biophys Res Commun, 2013; 440(1):105-11.

Jacobson TA, Ito MK, Maki KC, Orringer CE, Bays HE, Jones PH, McKenney JM, Grundy SM, Gill EA, Wild RA, Wilson DP, Brown WV. National lipid association recommendations for patient-centered management of dyslipidemia: full report. J Clin Lipidol, 2015; 9:129-69.

Katwan OJ, Alghamdi F, Almabrouk TA, Mancini SJ, Kennedy S, Oakhill JS, Scott JW, Salt IP. AMP-activated protein kinase complexes containing the beta2 regulatory subunit are up-regulated during and contribute to adipogenesis. Biochem J, 2019; 476(12):1725-40.

Khera AV, Emdin CA, Drake I, Natarajan P, Bick AG, Cook NR, Chasman DI, Baber U, Mehran R, Rader DJ, Fuster V, Boerwinkle E, Melander O, Orho-Melander M, Ridker PM, Kathiresan S. Genetic risk, adherence to a healthy lifestyle, and coronary disease. N Engl J Med, 2016; 375:2349-58

Koskinas KC, Siontis GCM, Piccolo R, Mavridis D, Räber L, Mach F, Windecker S. Effect of statins and non-statin LDL- lowering medications on cardiovascular outcomes in secondary prevention: a metaanalysis of randomized trials. Eur Heart J, 2018; 39:1172-80.

Lalwani ND, Hanselman JC, MacDougall DE, Sterling LR, Cramer CT. Complementary low-density lipoprotein-cholesterol lowering and pharmacokinetics of adding bempedoic acid (ETC-1002) to highdose atorvastatin background therapy in hypercholesterolemic patients: a randomized placebo-controlled trial. J Clin Lipidol, 2019; 13(4):568-79.

Laufs U, Banach M, Mancini GBJ, Gaudet D, Bloedon LT, Sterling LR, Kelly S, Stroes ESG. Efficacy and safety of bempedoic acid in patients with hypercholesterolemia and statin intolerance. J Am Heart Assoc, 2019; 8(7):e011662.

Lemus HN, Mendivil CO. Adenosine triphosphate citrate lyase: emerging target in the treatment of dyslipidemia. J Clin Lipidol, 2015; 9(3):384-9.

Marino G, Pietrocola F, Kong Y, Eisenberg T, Hill JA, Madeo F, Kroemer G. Dimethyl alpha-ketoglutarate inhibits maladaptive autophagy in pressure overload-induced cardiomyopathy. Autophagy, 2014; 10(5):930-2.

Martin SS, Joshi PH, Michos ED. Lipids in coronary heart disease: from epidemiology to therapeutics. In: Aronow WS, McClung JA, (eds.). Translational research in coronary artery disease (pp. 67-80). Boston, MA: Academic Press, 2016.

Molusky MM, Hsieh J, Lee SX, Ramakrishnan R, Tascau L, Haeusler RA, Accili D, Tall AR. Metformin and AMP kinase activation increase expression of the sterol transporters ABCG5/8 (ATP-binding cassette transporter G5/G8) with potential anti-atherogenic consequences. Arterioscler Thromb Vasc Biol, 2018; 38(7):1493-503.

Namgaladze D, Zukunft S, Schnutgen F, Kurrle N, Fleming I, Fuhrmann D, Brüne B. Polarization of human macrophages by interleukin-4 does not require ATP-citrate lyase. Front Immunol, 2018; 9:2858. 
Navarese EP, Robinson JG, Kowalewski M, Kolodziejczak M, Andreotti F, Bliden K, Tantry U, Kubica J, Raggi P, Gurbel PA. Association between baseline LDL-C level and total and cardiovascular mortality after LDL-C lowering: a systematic review and meta-analysis. JAMA, 2018; 319:1566-79.

Newman CB, Preiss D, Tobert JA, Jacobson TA, Page II RL, Goldstein LB, Chin C, Tannock LR, Miller M, Raghuveer G, Duell PB, Brinton EA, Pollak A, Braun LT, Welty FK, and on behalf of the American Heart Association Clinical Lipidology, Lipoprotein, Metabolism and Thrombosis Committee, a Joint Committee of the Council on Atherosclerosis, Thrombosis and Vascular Biology and Council on Lifestyle and Cardiometabolic Health; Council on Cardiovascular Disease in the Young; Council on Clinical Cardiology; and Stroke Council. Statin safety and associated adverse events: a scientific statement from the American Heart Association. Arterioscler Thromb Vasc Biol, 2019; 39(2):e38-81.

Nikolic D, Mikhailidis DP, Davidson MH, Rizzo M, Banach M. ETC-1002: a future option for lipid disorders? Atherosclerosis, 2014; 237(2):705-10.

O'Donoghue ML, Fazio S, Giugliano RP, Stroes ESG, Kanevsky E, Gouni-Berthold I, Im K, Pineda AL, Wasserman SM, Češka R, Ezhov MV, Jukema JW, Jensen HK, Tokgözoğlu SL, Mach F, Huber K, Sever PS, Keech AC, Pedersen TR, Sabatine MS. Lipoprotein(a), PCSK9 inhibi- tion, and cardiovascular risk: insights from the FOURIER trial. Circulation, 2019; 139:1483-92.

Oniciu DC, Dasseux JL, Yang J, Mueller R, Pop E, Denysenko A, Duan C, Huang TB, Zhang L, Krause BR, Drake SL, Lalwani N, Cramer CT, Goetz B, Pape ME, McKee A, Fici GJ, Lutostanski JM, Brown SC, Bisgaier CL. Influence of various central moieties on the hypolipidemic properties of long hydrocarbon chain diols and diacids. J Med Chem, 2006 49(1):334-48.

Pinkosky SL, Filippov S, Srivastava RA, Hanselman JC, Bradshaw CD, Hurley TR, Cramer CT, Spahr MA, Brant AF, Houghton JL, Baker C, Naples M, Adeli K, Newton RS. AMP-activated protein kinase and ATP-citrate lyase are two distinct molecular targets for ETC-1002, a novel small molecule regulator of lipid and carbohydrate metabolism. J Lipid Res, 2013; 54(1):134-51.

Pinkosky SL, Groot PHE, Lalwani ND, Steinberg GR. Targeting ATP-citrate lyase in hyperlipidemia and metabolic disorders. Trends Mol Med, 2017; 23(11):1047-63.

Pinkosky SL, Newton RS, Day EA, Ford RJ, Lhotak S, Austin RC, Birch CM, Smith BK, Filippov S, Groot PHE, Steinberg GR, Lalwani ND. Liver-specific ATP-citrate lyase inhibition by bempedoic acid decreases LDL-C and attenuates atherosclerosis. Nat Commun, 2016; 7:13457.

Ray KK, Bays HE, Catapano AL, Lalwani ND, Bloedon LT, Sterling LR, Robinson PL, Ballantyne CM, CLEAR Harmony Trial. Safety and efficacy of Bempedoic acid to reduce LDL cholesterol. N Engl J Med, 2019; 380(11):1022-32.

Rosenson RS, Baker S, Banach M, Borow KM, Braun LT, Bruckert E, Brunham LR, Catapano AL, Elam MB, Mancini GBJ, Moriarty PM, Morris PB, Muntner P, Ray KK, Stroes ES, Taylor BA, Taylor VH, Watts GF, Thompson PD. Optimizing cholesterol treatment in patients with muscle complaints. J Am Coll Cardiol, 2017; 70:1290-301.

Ruscica M, Banach M, Sahebkar A, Corsini A, Sirtori CR. ETC-1002 (Bempedoic acid) for the management of hyperlipidemia: from preclinical studies to phase 3 trials. Expert Opin Pharmacother, 2019; 20(7):791-803.

Samsoondar JR, Burke AC, Sutherland BG, Telford DE, Sawyez CG, Edwards JY, Pinkosky SL, Newton RS, Huff MW. Prevention of dietinduced metabolic dysregulation, inflammation, and atherosclerosis in Ldlr $(-/-)$ mice by treatment with the ATP-citrate Lyase inhibitor bempedoic acid. Arterioscler Thromb Vasc Biol, 2017; 37(4):647-56.
Sivanand S, Rhoades S, Jiang Q, Lee JV, Benci J, Zhang J, Yuan S, Viney I, Zhao S, Carrer A, Bennett MJ, Minn AJ, Weljie AM, Greenberg RA, Wellen KE. Nuclear acetyl-CoA production by ACLY promotes homologous recombination. Mol Cell, 2017; 67(2):252-65.

Thompson JF, Hyde CL, Wood LS, Paciga SA, Hinds DA, Cox DR, Hovingh GK, Kastelein JJP. Comprehensive whole-genome and candidate gene analysis for response to statin therapy in the Treating to New Targets (TNT) cohort. Circ Cardiovasc Genet, 2009; 2(2):173-81.

Thompson PD, MacDougall DE, Newton RS, Margulies JR, Hanselman JC, Orloff DG, McKenney JM, Ballantyne CM. Treatment with ETC-1002 alone and in combination with ezetimibe lowers LDL cholesterol in hypercholesterolemic patients with or without statin intolerance. J Clin Lipidol, 2016; 10(3):556-67.

Thompson PD, Rubino J, Janik MJ, MacDougall DE, McBride SJ, Margulies JR, Newton RS. Use of ETC-1002 to treat hypercholesterolemia in patients with statin intolerance. J Clin Lipidol, 2015; 9(3): 295-304.

Verschueren KHG, Blanchet C, Felix J, Dansercoer A, Vos DD, Bloch Y, Beeumen JV, Svergun D, Gutsche I, Savvides SN, Verstraete K. Structure of ATP citrate lyase and the origin of citrate synthase in the Krebs cycle. Nature, 2019; 568(7753):571-5.

Wang X, Luo S, Gan X, He C, Huang R. Safety and efficacy of ETC-1002 in hypercholesterolaemic patients: a meta-analysis of randomised controlled trials. Kardiol Pol, 2019; 77(2):207-16.

Wei J, Leit S, Kuai J, Therrien E, Rafi S, Harwood Jr HJ, DeLaBarre B, Tong L. An allosteric mechanism for potent inhibition of human ATP-citrate lyase. Nature, 2019; 568(7753):566-70.

Wellen KE, Hatzivassiliou G, Sachdeva UM, Bui TV, Cross JR, Thompson CB. ATP-citrate lyase links cellular metabolism to histone acetylation. Science (New York, NY), 2009; 324(5930):1076-80.

Xie S, Zhou F, Wang J, Cao H, Chen Y, Liu X, Zhang Z, Dai J, He X. Functional polymorphisms of ATP citrate lyase gene predicts clinical outcome of patients with advanced colorectal cancer. World J Surg Oncol, $2015 ; 13: 39-42$.

Xu J, Yin L, Xu Y. Li Y, Zalzala M, Cheng G, Zhang Y. Hepatic carboxylesterase 1 is induced by glucose and regulates postprandial glucose levels. PLoS One, 2014; 9(10):e109663.

Zagelbaum NK, Yandrapalli S, Nabors C, Frishman WH Bempedoic acid (ETC- 1002): ATP citrate Lyase inhibitor: review of a firstin-class medication with potential benefit in statin-refractory cases. Cardiol Rev, 2019; 27(1):49-56.

Zhao S, Torres A, Henry RA, Trefely S, Wallace M, Lee JV, Carrer A, Sengupta A, Campbell SL, Kuo YM, Frey AJ, Meurs N, Viola JM, Blair IA, Weljie AM, Metallo CM, Snyder NW, Andrews AJ, Wellen KE. ATP-citratelyase controls a glucose-to-acetate metabolic switch. Cell Rep, 2016; 17(4):1037-52.

\section{How to cite this article:}

Korzh O. Defining the role of bempedoic acid in lowering low-density lipoprotein cholesterol. J Appl Pharm Sci, 2020; 10(12):130-139. 